\title{
Generation Process and Sterilization Effect of OH Radical in a Steam Plasma Flow at Atmospheric Pressure for a Plasma Autoclave
}

\author{
Takehiko Sato $^{1, *} \&$ Takeshi Furui ${ }^{2}$ \\ ${ }^{1}$ Institute of Fluid Science, Tohoku University, Sendai, Japan; ${ }^{2}$ Graduate School of Engineering, \\ Tohoku University, Sendai, Japan (Currently, Santo Co. Ltd., Yokkaichi, Japan) \\ *Address all correspondence to: Takehiko Sato, Institute of Fluid Science, Tohoku University, 2-1-1 Katahira, Aoba-ku, \\ Sendai 980-8577, Japan; sato@ifs.tohoku.ac.jp
}

\begin{abstract}
The reactive flow field and the mechanism of sterilization by steam plasma flow at atmospheric pressure were clarified to develop a new sterilization system by experimental and computational methods. The emission lines of $\mathrm{OH}$, whose intensity increased with increased applied voltage and frequency, were observed in the discharge region. The $\mathrm{OH}$ radical finally changed to $\mathrm{H}_{2} \mathrm{O}_{2}$ in the condensed water. The $\mathrm{OH}$ radical was capable of sterilizing spores of Geobacillus stearothermophilus, although the condensed water did not have such effect. The discharge process was clarified with an axisymmetric, non-Maxwellian model. The $\mathrm{OH}$ radical was generated by a wire electrode toward the ground electrode, and the concentration of $\mathrm{H}_{2} \mathrm{O}_{2}$ increased after streamer propagation.
\end{abstract}

KEY WORDS: dielectric barrier discharges (DBD), sterilization, computational analysis

\section{INTRODUCTION}

Infectious diseases caused by pathogenic microorganisms pose a widespread problem. To prevent such infection, a safe and low-cost sterilization system needs to be developed. The autoclave has been the main method for sterilizing medical instruments because of its simple configuration and low-cost operation. However, this method is not suitable for low-heat-resistant materials such as polymers, which are increasingly employed in modern medical instruments. Therefore, it is necessary to establish an advanced sterilization method that can be operated at lower temperature.

To decrease the operating temperature, sterilization methods using low-temperature plasma flow at atmospheric pressure have been developed. ${ }^{1-4}$ There are several methods to produce such plasma, including direct current discharge, radio-frequency discharge, dielectric barrier discharge (DBD) and pulsed corona discharge. For example, Shimizu et al. ${ }^{5}$ have applied a microwave argon plasma flow, which generates toxic by-products at a safe level, to treat dermatoses. Sladek and Stoffels ${ }^{6}$ have developed a plasma needle, which has been reported in many papers, using helium gas for plasma treatment; and Weltmann et al. ${ }^{7}$ have also developed atmospheric pressure plasma jets (APPJs) for antimicrobial treatment. Deng et al. ${ }^{8}$ have sterilized bacteria using atmospheric pressure glow discharges with helium gas. Furthermore, Moisan et al. ${ }^{9}$ have also clarified 
the effectiveness of chemically active species for sterilization. Laroussi and Leipold ${ }^{10}$ have reported the effects of UV light, heat, and reactive species on the inactivation of bacteria; and Kim et al. ${ }^{11}$ have applied species generated with atmospheric air plasma to kill cancer cells. Sato et al. ${ }^{12,13}$ have reported the sterilization mechanism of Escherichia coli by chemical species.

We have previously reported the sterilization efficacy and mechanism of a tube using DBD. ${ }^{14,15}$ This system consisted of an insulated wire electrode set in the tube and a flat grounded electrode set below the tube. It was possible to sterilize spores of Geobacillus stearothermophilus by such treatment for 5 minutes around $70^{\circ} \mathrm{C}$. The main sterilization factors were transport and concentration of chemical species such as nitrogen oxide and reactive oxygen species by a twin vortex induced by plasma.

In our previous studies, this plasma sterilization system was applied to a pure steam flow operated at $100^{\circ} \mathrm{C}$ and atmospheric pressure to develop an innovative autoclave. The plasma source consisted of a stainless steel wire installed in a quartz tube with diameter of $8 \mathrm{~mm}$ and a grounded electrode located at the outer wall of the tube. The flow rate of the steam at atmospheric pressure in the tube is $8.1 \mathrm{~L} / \mathrm{min}$, which is equal to a velocity of $2.7 \mathrm{~m} / \mathrm{s}$. When the applied voltage was $13 \mathrm{kV}$ peak-to-peak and its frequency was $3 \mathrm{kHz}$, the highest efficacy of sterilization against $G$. stearothermophilus spores was obtained in the voltage range from 11 to $14 \mathrm{kV}$ peak-to-peak and the frequency range from 1 to $5 \mathrm{kHz}$. Complete sterilization required $50 \mathrm{~min}$, and partial sterilization required 30 min. ${ }^{16}$ However, the sterilization mechanism in the pure steam plasma flow is not yet fully understood.

The objective of the present study was to clarify experimentally and computationally the reactive flow field and the mechanism of sterilization by a steam plasma flow at atmospheric pressure in order to develop a new sterilization system.

\section{EXPERIMENTAL PART}

\section{A. Methods}

Figure 1 shows a schematic of the experimental apparatus (a) and details of the discharge reactor for a straight type (b) and a branch type (c). Pure steam is generated by boiling purified water in a flask with a $250-\mathrm{W}$ electric heater. The average volume flow rate of generated steam was $8.1 \mathrm{~L} / \mathrm{min}$, which equals a velocity of $2.7 \mathrm{~m} / \mathrm{s}$ in a quartz tube with an inner diameter of $8 \mathrm{~mm}$, an outer diameter of $10 \mathrm{~mm}$, and a length of $155 \mathrm{~mm}$. A stainless steel wire electrode (SUS304), $0.28 \mathrm{~mm}$ in diameter, was set in the quartz tube, and a grounded aluminum electrode $2 \mathrm{~mm}$ in width and $100 \mathrm{~mm}$ in length was set on the outer wall of the quartz tube. The configuration of the grounded electrode changed from the previously reported configuration ${ }^{16}$ to enable direct analysis of the region. This change resulted in a sterilization effect different from that previously reported. ${ }^{16} \mathrm{~A}$ high peak-to-peak voltage of $16 \mathrm{kV}$ with a frequency of $3 \mathrm{kHz}$ by a high voltage amplifier (HVA4321, NF Corp., Japan) generated the plasma flow. Water condensed by the steam plasma flow using a cooling system was collected. When $\mathrm{OH}$ increased, air from a gas 


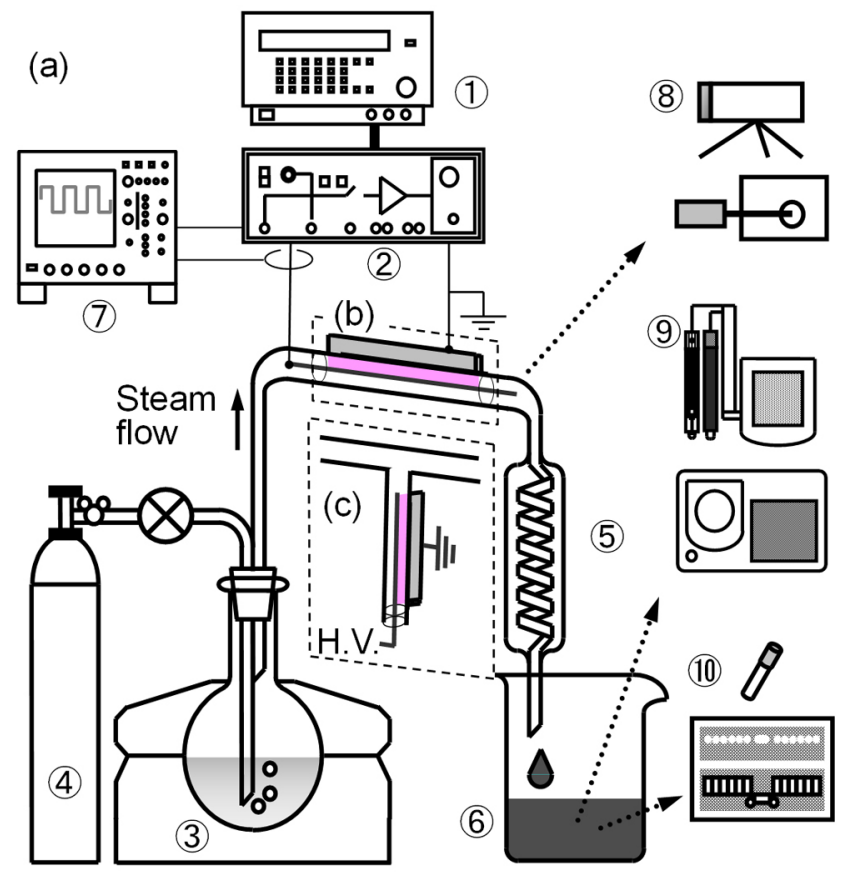

1. Power supply

2. Discharge tube

3. Steam generator system

4. Gas cylinder

5. Condenser
6. Condensed water

7. Oscilloscope

8. Optical system

9. Water quality meters

10. Sterilization check device

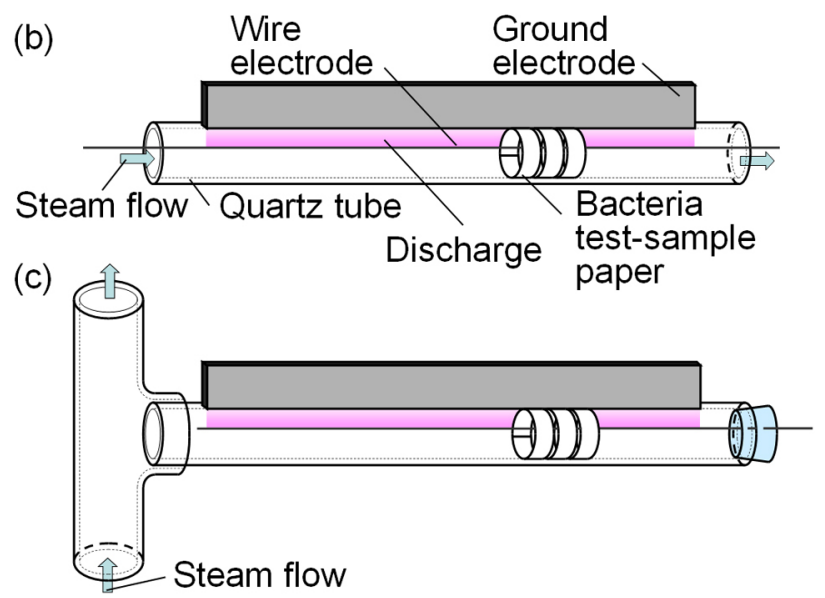

FIG. 1: Schematic of experimental apparatus (a) and details of the straight-type (b) and branch-type (c) tubes. 
cylinder was bubbled through the purified water and fed into the steam.

The discharge current $I_{d}$ and the applied voltage $V_{d}$ were measured by a digital oscilloscope (Wave Runner 62Xi, LeCroy, France). A current probe (AP015, LeCroy, France) was used for measuring the current of the cable that supplied the wire electrode. The average discharge power $P_{d}$ was obtained by the equation.$^{17}$

Side view photographs were taken with a digital camera (Nikon, D50). Spectrum photographs of the discharge region in the cross section were taken with a highly sensitive UV camera (C9299-02, Hamamatsu Photonics, Japan) using a bandpass filter with a center wavelength of $310 \mathrm{~nm}$ and a full width at half maximum (FWHM) of $10 \mathrm{~nm}$ (Andover, USA). The spectrum from 200 to $860 \mathrm{~nm}$ was measured by a high sensitivity optical multichannel analyzer (PMA-12, Hamamatsu Photonics, Japan), because the emission intensity from the discharge region was weak. ${ }^{18}$

The concentration of $\mathrm{H}_{2} \mathrm{O}_{2}$ in the condensed water was measured with a digital water quality meter (DPM-MT, Kyoritsu Chemical-Check Lab., Japan) and a reagent (WAK- $\mathrm{H}_{2} \mathrm{O}_{2} /-\mathrm{H}_{2} \mathrm{O}_{2}(\mathrm{C})$, Kyoritsu Chemical-Check Lab., Japan). The $\mathrm{pH}$ and oxidation reduction potential (ORP) as measured by a digital pH/ORP meter (PC6500, Eutech Instruments, USA) have resolutions of $0.001 \mathrm{pH}$ and $0.1 \mathrm{mV}$, respectively. ${ }^{19}$

The sterilization test was performed using a biological indicator (Attest Auto Reader 290, 3M), which evaluates spores of G. stearothermophilus (ATCC 7953) as reference bacteria. The bacteria were not sterilized at $100^{\circ} \mathrm{C}$ for 180 minutes. As shown in Figure 1, three bacteria test sample papers, $25 \mathrm{~mm}$ long and $5 \mathrm{~mm}$ wide, were set along the wall circumferentially for each sterilization condition. A negative sign (-) means that spores of $G$. stearothermophilus were sterilized by at least a log reduction number of $5\left(10^{-5}\right)$. A positive sign $(+)$ means that the log reduction number was less than $5 .{ }^{20}$

To clarify the effect of gas velocity on sterilization, we reduced the gas velocity by setting the tube normal to the main flow tube, as shown in Figure 1 (c). The quartz tube was surrounded by a heat insulator. The effect of the condensed water on sterilization was tested by immersing the test samples in the condensed water.

\section{B. Experimental Results and Discussion}

Figure 2 (a) shows waveforms of the discharge current and the applied peak-to-peak voltage of $16 \mathrm{kV}$ with a frequency of $4 \mathrm{kHz}$. When the applied peak-to-peak voltage was greater than $12 \mathrm{kV}$, pulse currents were observed. When the pulse currents appeared, radiation was also observed, as shown in Figure 2 (b). The radiation region was distributed along the wire electrode and extended towards the grounded electrode from the wire electrode. The radiation intensity rose with the increase of the applied voltage and the frequency as shown in Figure 3 (a) and (b), respectively. These results of the optical emission spectroscopy imply the generation of $\mathrm{OH}$ with wavelengths of $282.9 \mathrm{~nm}, 289.3$ $\mathrm{nm}$, and $308.9 \mathrm{~nm}$ in the steam plasma flow. ${ }^{21}$ There is a small peak of H $\alpha$ with a wavelength of $656.2 \mathrm{~nm},{ }^{22}$ although background noise increased with increasing wavelength.

The two-dimensional profiles of emission intensity are shown in Figure 4, for case (a) without filter, and case (b) with a bandpass filter of $310 \mathrm{~nm}$ corresponding to $\mathrm{OH}$ 


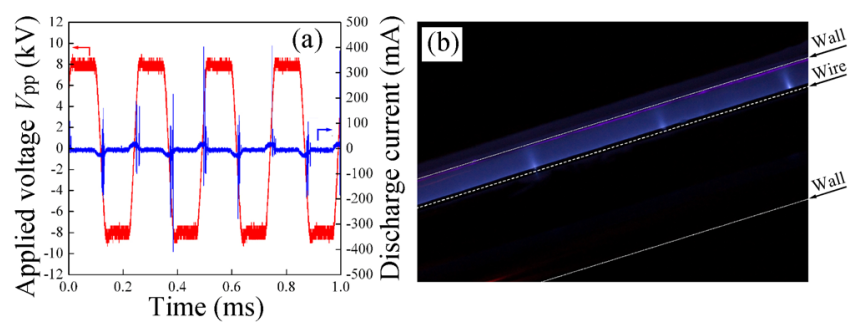

FIG. 2: (a) Waveforms of discharge current and applied peak-to-peak voltage of $16 \mathrm{kV}$ with a frequency of $4 \mathrm{kHz}$. (b) Side view photograph of the discharge region at $V_{\mathrm{pp}}=16$ $\mathrm{kV}$.

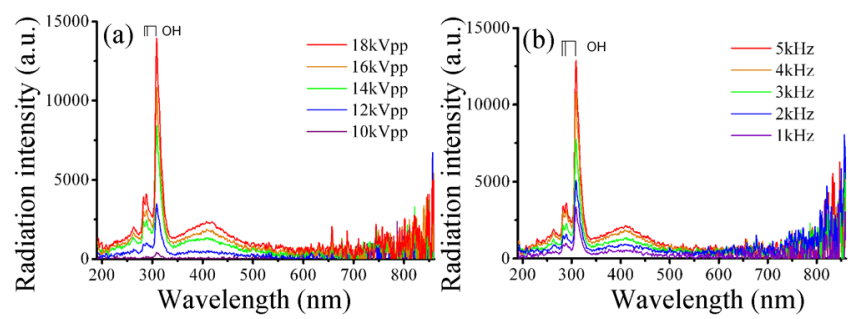

FIG. 3: Optical emission spectroscopy for (a) different applied voltages at $f=4 \mathrm{kHz}$ and (b) different frequencies at $V_{\mathrm{pp}}=16 \mathrm{kV}$.

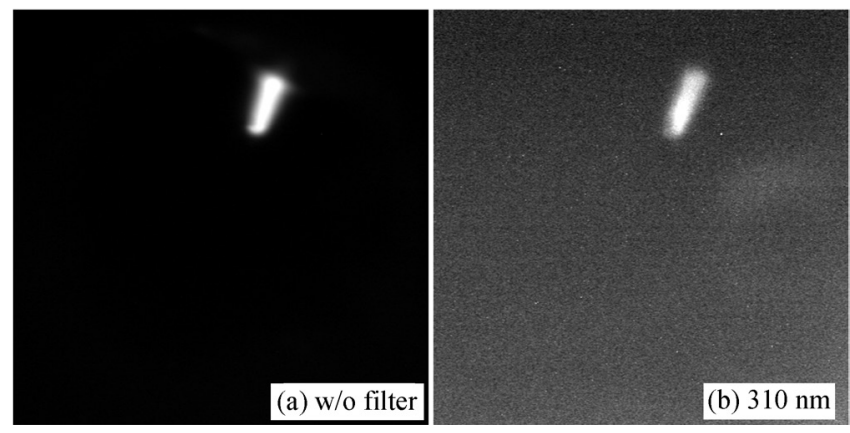

FIG. 4: Two-dimensional profiles of emission intensity. (a) Without filter. (b) Through the band-pass filter of $310 \mathrm{~nm}$.

emission. The bright region between the wire and the ground electrodes is large because the discharge occurs in this region. Although the greater intensity of $\mathrm{OH}$ emission is distributed near the wire electrode, which is different from the result obtained when a filter is absent.

Figure 5 (a) shows the $\mathrm{H}_{2} \mathrm{O}_{2}$ concentration against the applied voltage for different frequencies. The concentration increased to greater than $30 \mathrm{mg} / \mathrm{L}$ at a peak-to-peak voltage of $18 \mathrm{kV}$ with a frequency of $4 \mathrm{kHz}$ and then increasing voltage or frequency. 

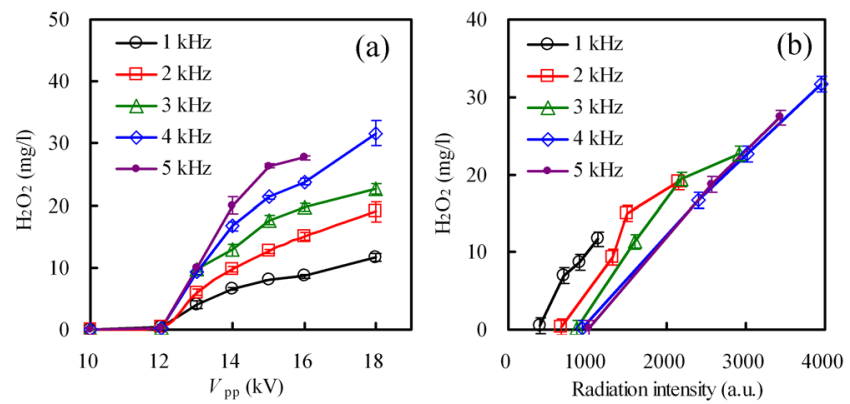

FIG. 5: (a) $\mathrm{H}_{2} \mathrm{O}_{2}$ concentration against the applied voltage for different frequencies. (b) Relationship between the intensity of $\mathrm{OH}$ and $\mathrm{H}_{2} \mathrm{O}_{2}$ concentration.
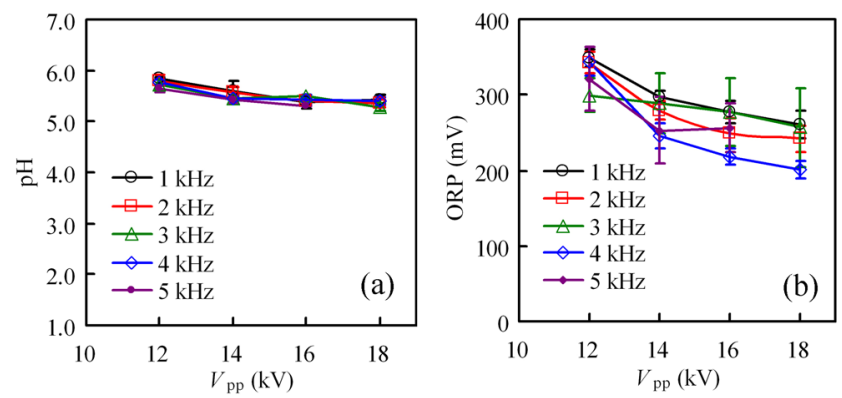

FIG. 6: (a) pH value and (b) ORP against the applied voltage.

The $\mathrm{H}_{2} \mathrm{O}_{2}$ concentration was proportional to the 308.9-nm intensity of $\mathrm{OH}$, as shown in Figure 5 (b). This result means that the generated $\mathrm{OH}$ finally changes to $\mathrm{H}_{2} \mathrm{O}_{2}$. However, the characteristics of the $\mathrm{pH}$ value and ORP against the applied voltage were slightly decreased, as shown in Figures 6 (a) and (b), respectively, but their tendencies were not congruent with $\mathrm{H}_{2} \mathrm{O}_{2}$ concentration. Therefore, most of the generated $\mathrm{H}$, which was generated in the process of $\mathrm{OH}$ generation, was possibly changed to a stable compound such as $\mathrm{H}_{2}$ gas, because those values are affected by the $\mathrm{H}$ concentration in water.

Table 1 shows the sterilization efficacy (a) with a gas flow of $2.7 \mathrm{~m} / \mathrm{s}$ using a straighttype tube, and (b) under the low-gas flow velocity using the branch-type tube, as shown in Figure 1 (b) and (c), respectively. For the straight-type tube, one test sample was sterilized under the condition of $V_{p p}=16 \mathrm{kV}, f=3 \mathrm{kHz}$, and sterilization time $t=40 \mathrm{~min}$. When the applied voltage was increased to $V_{p p}=18 \mathrm{kV}$, two test samples were sterilized. This result implies that the $\mathrm{OH}$ is an important sterilization factor, ${ }^{3,4}$ because the intensity of the $\mathrm{OH}$ emission line and the concentration of $\mathrm{H}_{2} \mathrm{O}_{2}$ increased correspondingly. For the branch-type tube, two test samples were also sterilized under the condition of $V_{p p}=16 \mathrm{kV}, f=3 \mathrm{kHz}$, and sterilization time $t=40 \mathrm{~min}$. Thus, three test samples were not sterilized within $40 \mathrm{~min}$ in both types of tubes. To increase $\mathrm{OH}$ generation, air was mixed with the steam flow, results of its optical emission spectroscopy are shown in 
TABLE 1: Sterilization efficacy (a) under the gas flow of $2.7 \mathrm{~m} / \mathrm{s}$ using straight-type tube, and (b) under the low-gas flow velocity using branch-type tube.

\begin{tabular}{llll}
\hline $\begin{array}{l}\text { (a) Straight-type tube } \\
\begin{array}{l}\text { Applied voltage } \\
\boldsymbol{V}(\mathbf{k V})\end{array}\end{array}$ & $\begin{array}{l}\text { Frequency } \\
\boldsymbol{f}(\mathbf{k H z})\end{array}$ & $\begin{array}{l}\text { Sterilization time } \\
\boldsymbol{t}_{\mathbf{s}}(\mathbf{m i n})\end{array}$ & $\begin{array}{l}\text { Sterilization } \\
\text { result }\end{array}$ \\
\hline 16 & 3 & 30 & ++- \\
16 & 3 & 40 & ++- \\
18 & 3 & 40 & +-- \\
\hline
\end{tabular}

(b) Branch-type tube

\begin{tabular}{llll}
$\begin{array}{l}\text { Applied voltage } \\
\boldsymbol{V}(\mathbf{k V})\end{array}$ & $\begin{array}{l}\text { Frequency } \\
\boldsymbol{f}(\mathbf{k H z})\end{array}$ & $\begin{array}{l}\text { Sterilization time } \\
\boldsymbol{t}_{\boldsymbol{s}}(\mathbf{m i n})\end{array}$ & $\begin{array}{l}\text { Sterilization } \\
\text { result }\end{array}$ \\
\hline 16 & 3 & 10 & +++ \\
16 & 3 & 20 & +++ \\
16 & 3 & 30 & ++- \\
16 & 3 & 40 & +-- \\
\hline
\end{tabular}

Figure 7. The emission intensity of the $\mathrm{OH}$ line increased with increasing air flow rate. The intensities of the $\mathrm{N}_{2}$ second positive system also increased. The sterilization efficacy under the air mixing condition at $V_{p p}=16 \mathrm{kV}$ and $f=3 \mathrm{kHz}$ is shown in Table 2. Three test samples were sterilized for 30 minutes under air flow rates of 1 and $4 \mathrm{~L} / \mathrm{min}$, although sample sterilization was not achieved under a rate of $8 \mathrm{~L} / \mathrm{min}$.

\section{COMPUTATIONAL PART}

\section{A. Computational Model}

Figure 8 shows the computational domain. It was a two-dimensional axisymmetric model since the streamer is considered to be a filament rather than a plane configuration. The quartz tube had an inner diameter of $8 \mathrm{~mm}$ and an outer diameter of $10 \mathrm{~mm}$. The wire electrode with a diameter of $0.28 \mathrm{~mm}$ was set at the center of the tube. Computational analysis was performed with the commercial software program CFD-ACE+ (ESI-Group).

\section{B. Assumptions}

We determined that the $\mathrm{OH}$ was generated in the plasma from water vapor and later changed to $\mathrm{H}_{2} \mathrm{O}_{2}$. Computational analysis was performed assuming the following conditions: (1) two-dimensional axisymmetry; (2) three fluids (electrons, ions, and neutral particles) and two temperatures (electrons and heavy particles); (3) non-Maxwellian distribution for electron energy; (4) unsteady, laminar flow and perfect gas; (4) dissociation, ionization, recombination, and electron attachment; (5) presence of surface 


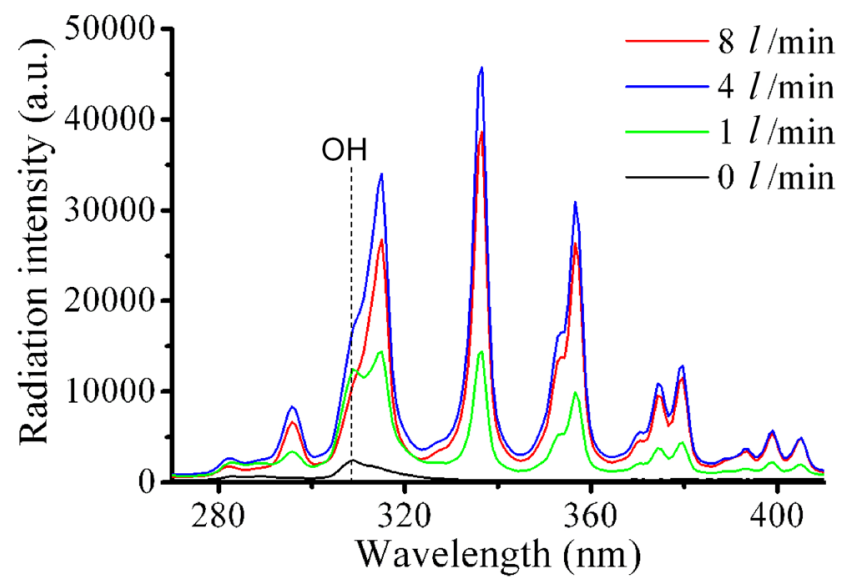

FIG. 7: Optical emission spectroscopy in the case of mixing of air in the steam flow.

TABLE 2: Sterilization efficacy under the air mixing condition at $V_{p p}=16 \mathrm{kV}, f=3 \mathrm{kHz}$.

\begin{tabular}{lll}
\hline $\begin{array}{l}\text { Flow rate } \\
\boldsymbol{Q}_{\text {air }}(\text { L/min })\end{array}$ & $\begin{array}{l}\text { Sterilization time } \\
\boldsymbol{t}(\mathrm{min})\end{array}$ & $\begin{array}{l}\text { Sterilization } \\
\text { result }\end{array}$ \\
\hline 0.1 & 15 & +++ \\
1 & 15 & ++- \\
4 & 15 & +-- \\
8 & 15 & ++- \\
0.1 & 30 & +++ \\
1 & 30 & --- \\
4 & 30 & --- \\
8 & 30 & +++ \\
\hline
\end{tabular}

charge on the inner wall; and (6) absence of radiation loss, photoionization, gravity and condensation of water vapor.

\section{Governing Equations}

The governing equations included conversation of mass, momentum, energy, and chemical species; state of equation, and Poisson's equation. The flow, heat, chemical, plasma, and electromagnetic modules of CFD-ACE $+{ }^{35}$ were used for solving the governing equations. The electron distribution was derived by the Fokker-Planck equation in the plasma module.

The following 23 chemical species were adopted in this model: $\mathrm{H}^{+}, \mathrm{H}^{-}, \mathrm{H}_{2}^{+}, \mathrm{H}_{2} \mathrm{O}^{+}$, $\mathrm{H}_{3}^{+}, \mathrm{HO}_{2}^{+}, \mathrm{O}^{+}, \mathrm{O}^{-}, \mathrm{O}_{2}^{+}, \mathrm{O}_{2}^{-}, \mathrm{O}_{3}^{-}, \mathrm{OH}^{+}, \mathrm{OH}^{-}, \mathrm{H}, \mathrm{H}_{2}, \mathrm{H}_{2} \mathrm{O}, \mathrm{H}_{2} \mathrm{O}_{2}, \mathrm{HO}_{2}, \mathrm{O}, \mathrm{O}_{2}, \mathrm{O}_{3}, \mathrm{OH}$, and 


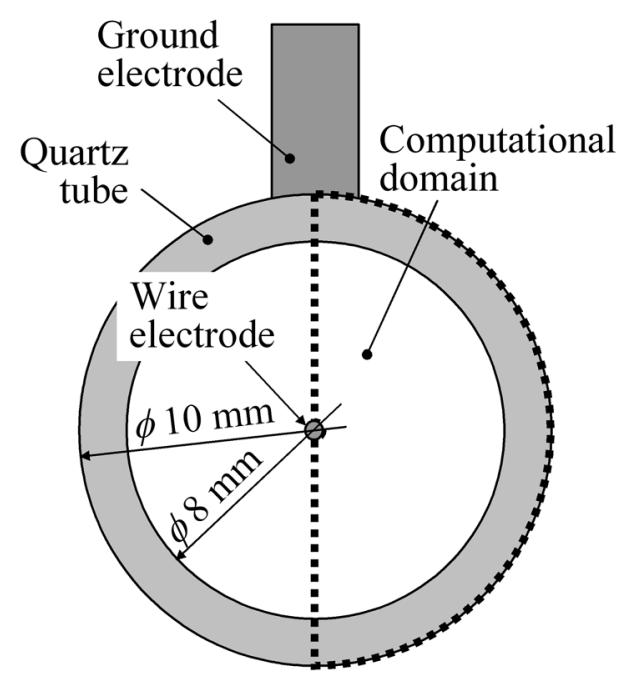

FIG. 8: Computational domain.

electrons. Sixteen electron collision reactions by using electron collision cross section data and 86 chemical reaction equations were considered, as shown in Table 3.

\section{Initial and Boundary Conditions}

All species were assumed to have velocity $u=0 \mathrm{~m} / \mathrm{s}$, temperature of heavy particles $T_{\mathrm{h}}$ $=373 \mathrm{~K}$, electron temperature $T_{\mathrm{e}}=0.04 \mathrm{eV}$, and potential $\varphi=0 \mathrm{kV}$. Mass fractions of chemical species were $Y_{H}=Y_{H 2}=Y_{H 2 O 2}=Y_{H O 2}=Y_{O}=Y_{O 2}=Y_{O 3}=Y_{O H}=10^{-8}$ for neutral particles; $Y_{H_{-}}=Y_{\mathrm{O}-}=Y_{\mathrm{O}_{2}-}=Y_{\mathrm{O} 3-}=Y_{\mathrm{OH}-}=10^{-12}$ for negative particles; and $Y_{\mathrm{H}^{+}}=Y_{\mathrm{H}_{2+}}=$ $Y_{\mathrm{H} 3+}=Y_{\mathrm{H} 2 \mathrm{O}+}=Y_{\mathrm{HO}^{2+}}=Y_{\mathrm{O}^{+}}=Y_{\mathrm{O}^{2+}}=Y_{\mathrm{OH}^{+}}=10^{-11}$ for positive particles. Electron number density was derived from $n_{e}=\sum_{i=i o n} q_{i} n_{i}$. Total mass fraction of all species $Y_{0}$ except $\mathrm{H}_{2} \mathrm{O}$ was derived from $Y_{H 2 O}=1-Y_{0}$.

Figure 9 shows the boundary conditions. The surface temperature of the wire electrode was constant at $100^{\circ} \mathrm{C}$. The outer wall of the quartz tube was adiabatic.

\section{E. Computational Results and Discussion}

Figure 10 shows the time and spatial evolutions of (a) number density of $\mathrm{OH}$ and (b) number density of $\mathrm{H}_{2} \mathrm{O}_{2}$. The cross section of the tube wall is dark gray. When the positive applied voltage increased to $t=0.8 \mu \mathrm{s}$, the strength of the electric field and the electron temperature increased around the central axis. Correspondingly, the electrons gathered here.

At $t=0.8 \mu \mathrm{s}$, the $\mathrm{OH}$ is generated around the wire electrode, then a high-density region of $\mathrm{OH}$ started to propagate toward the ground electrode. At $t=0.82 \mu \mathrm{s}$, the $\mathrm{OH}$ reached and spread widely along the upper wall surface. At $t=0.90 \mu \mathrm{s}$, the density 
TABLE 3: Electron collision reactions by using electron collision cross section data and chemical reaction equations.

\begin{tabular}{|c|c|c|c|}
\hline No. & Chemical reaction & $\begin{array}{l}\text { Rate constants } \\
\left(\mathrm{m}^{3}-\mathrm{kmol}^{-1}-\mathrm{s}^{-1}\right)\end{array}$ & Ref. \\
\hline 1 & $\mathrm{e}+\mathrm{H}_{2} \mathrm{O} \rightarrow \mathrm{e}+\mathrm{H}_{2} \mathrm{O}$ & collision cross section data & 23 \\
\hline 2 & $\mathrm{e}+\mathrm{H}_{2} \mathrm{O} \rightarrow 2 \mathrm{e}+\mathrm{H}_{2} \mathrm{O}^{+}$ & collision cross section data & 23 \\
\hline 3 & $\mathrm{e}+\mathrm{H}_{2} \mathrm{O} \rightarrow 2 \mathrm{e}+\mathrm{OH}^{+}+\mathrm{H}$ & collision cross section data & 23 \\
\hline 4 & $\mathrm{e}+\mathrm{H}_{2} \mathrm{O} \rightarrow 2 \mathrm{e}+\mathrm{O}^{+}+\mathrm{H}_{2}$ & collision cross section data & 23 \\
\hline 5 & $\mathrm{e}+\mathrm{H}_{2} \mathrm{O} \rightarrow 2 \mathrm{e}+\mathrm{H}_{2}^{+}+\mathrm{O}$ & collision cross section data & 23 \\
\hline 6 & $\mathrm{e}+\mathrm{H}_{2} \mathrm{O} \rightarrow 2 \mathrm{e}+\mathrm{H}^{+}+\mathrm{OH}$ & collision cross section data & 23 \\
\hline 7 & $\mathrm{e}+\mathrm{H}_{2} \mathrm{O} \rightarrow \mathrm{H}^{-}+\mathrm{OH}$ & collision cross section data & 23 \\
\hline 8 & $\mathrm{e}+\mathrm{H}_{2} \mathrm{O} \rightarrow \mathrm{O}^{-}+\mathrm{H}_{2}$ & collision cross section data & 23 \\
\hline 9 & $\mathrm{e}+\mathrm{H}_{2} \mathrm{O} \rightarrow \mathrm{OH}^{-}+\mathrm{H}$ & collision cross section data & 23 \\
\hline 10 & $\mathrm{e}+\mathrm{H}_{2} \mathrm{O} \rightarrow \mathrm{e}+\mathrm{OH}+\mathrm{H}$ & collision cross section data & 23 \\
\hline 11 & $\mathrm{e}+\mathrm{H} \rightarrow 2 \mathrm{e}+\mathrm{H}^{+}$ & collision cross section data & 24 \\
\hline 12 & $\mathrm{e}+\mathrm{H} \rightarrow 2 \mathrm{e}+\mathrm{H}$ & collision cross section data & 24 \\
\hline 13 & $\mathrm{e}+\mathrm{H} \rightarrow 3 e+\mathrm{H}^{+}$ & collision cross section data & 24 \\
\hline 14 & $\mathrm{e}+\mathrm{O} \rightarrow 2 \mathrm{e}+\mathrm{O}^{+}$ & collision cross section data & 24 \\
\hline 15 & $e+O \rightarrow 2 e+O$ & collision cross section data & 24 \\
\hline 16 & $\mathrm{e}+\mathrm{O} \rightarrow 3 \mathrm{e}+\mathrm{O}^{+}$ & collision cross section data & 24 \\
\hline 17 & $\mathrm{e}+\mathrm{O}_{3} \rightarrow \mathrm{O}-+\mathrm{O}_{2}$ & $3.0 \times 10^{-15}$ & \\
\hline 18 & $\mathrm{e}+\mathrm{O}_{3} \rightarrow \mathrm{O}_{2}^{-}+\mathrm{O}$ & $1.3 \times 10^{-33}$ & \\
\hline 19 & $\mathrm{e}+\mathrm{H}_{2} \mathrm{O}^{+} \rightarrow \mathrm{OH}+\mathrm{H}$ & $6.6 \times 10^{-9} T_{e}^{-0.5}$ & 33 \\
\hline 20 & $\mathrm{e}+\mathrm{H}_{2} \mathrm{O}^{+} \rightarrow \mathrm{O}+2 \mathrm{H}$ & $2.88 \times 10^{-9} T_{e}^{-0.5}$ & 33 \\
\hline 21 & $\mathrm{e}+\mathrm{H}_{2} \mathrm{O}^{+} \rightarrow \mathrm{O}+\mathrm{H}_{2}$ & $2.52 \times 10^{-9} T_{e}^{-0.5}$ & 33 \\
\hline 22 & $\mathrm{e}+\mathrm{H}_{2}^{+} \rightarrow \mathrm{H}_{2}$ & $2.0 \times 10^{-10} T_{e}^{e}-0.5$ & \\
\hline 23 & $\mathrm{O}+\mathrm{O}_{3} \rightarrow 2 \mathrm{O}_{2}$ & $8.0 \times 10^{-15} \exp (-2060 / T)$ & \\
\hline 24 & $\mathrm{O}+\mathrm{OH} \rightarrow \mathrm{O}_{2}+\mathrm{H}$ & $2.4 \times 10^{-14} \exp (110 / T)$ & 25 \\
\hline 25 & $\mathrm{O}+\mathrm{HO}_{2} \rightarrow \mathrm{OH}+\mathrm{O}_{2}$ & $2.7 \times 10^{-14} \exp (224 / T)$ & 25 \\
\hline 26 & $\mathrm{O}+\mathrm{H}_{2} \mathrm{O}_{2} \rightarrow \mathrm{OH}+\mathrm{HO}_{2}$ & $1.4 \times 10^{-15} \exp (-2000 / T)$ & 25 \\
\hline 27 & $\mathrm{OH}+\mathrm{H}_{2} \rightarrow \mathrm{H}_{2} \mathrm{O}+\mathrm{H}$ & $7.7 \times 10^{-15} \exp (-2100 / T)$ & 25 \\
\hline 28 & $\mathrm{OH}+\mathrm{HO}_{2} \rightarrow \mathrm{H}_{2} \mathrm{O}+\mathrm{O}_{2}$ & $4.8 \times 10^{-14} \exp (250 / T)$ & 25 \\
\hline 29 & $\mathrm{OH}+\mathrm{H}_{2} \mathrm{O}_{2} \rightarrow \mathrm{H}_{2} \mathrm{O}+\mathrm{HO}_{2}$ & $2.9 \times 10^{-15} \exp (-160 / T)$ & 25 \\
\hline 30 & $\mathrm{OH}+\mathrm{O}_{3} \rightarrow \mathrm{HO}_{2}+\mathrm{O}_{2}$ & $1.7 \times 10^{-15} \exp (-940 / T)$ & 25 \\
\hline 31 & $2 \mathrm{HO}_{2} \rightarrow \mathrm{H}_{2} \mathrm{O}_{2}+\mathrm{O}_{2}$ & $2.2 \times 10^{-16} \exp (600 / T)$ & 25 \\
\hline 32 & $2 \mathrm{H}+\mathrm{H} \rightarrow \mathrm{H}_{2}+\mathrm{H}^{2}$ & $8.8 \times 10^{-39}$ & 26 \\
\hline 33 & $2 \mathrm{H}+\mathrm{H}_{2} \rightarrow \mathrm{H}_{2}+\mathrm{H}_{2}$ & $2.8 \times 10^{-37} T^{-0.6}$ & 26 \\
\hline 34 & $2 \mathrm{H}+\mathrm{H}_{2} \mathrm{O} \rightarrow \mathrm{H}_{2}+\mathrm{H}_{2} \mathrm{O}$ & $2.8 \times 10^{-35} T^{-1.0}$ & 26 \\
\hline
\end{tabular}


TABLE 3: (continued)

\begin{tabular}{|c|c|c|c|}
\hline No. & Chemical reaction & $\begin{array}{l}\text { Rate constants } \\
\left(\mathrm{m}^{3}-\mathrm{kmol}^{-1}-\mathbf{s}^{-1}\right)\end{array}$ & Ref. \\
\hline 35 & $\mathrm{H}+\mathrm{HO}_{2} \rightarrow \mathrm{H}_{2}+\mathrm{O}_{2}$ & $4.2 \times 10^{-14} \exp (-350 / T)$ & 27 \\
\hline 36 & $\mathrm{H}+\mathrm{HO}_{2} \rightarrow 2 \mathrm{OH}$ & $4.2 \times 10^{-13} \exp (-950 / T)$ & 27 \\
\hline 37 & $\mathrm{H}+\mathrm{HO}_{2} \rightarrow \mathrm{H}_{2} \mathrm{O}+\mathrm{O}$ & $8.3 \times 10^{-14} \exp (-500 / T)$ & 27 \\
\hline 38 & $\mathrm{HO}_{2}+\mathrm{O}_{3} \rightarrow \mathrm{OH}+2 \mathrm{O}_{2}$ & $1.4 \times 10^{-17} \exp (-600 / T)$ & 27 \\
\hline 39 & $\mathrm{H}+\mathrm{O}_{3} \rightarrow \mathrm{OH}+\mathrm{O}_{2}$ & $1.2 \times 10^{-13} \exp (-560 / T)$ & 27 \\
\hline 40 & $\mathrm{O}+\mathrm{H}_{2} \rightarrow \mathrm{OH}+\mathrm{H}$ & $1.6 \times 10^{-14} \exp (-4570 / T)$ & 28 \\
\hline 41 & $\mathrm{H}+\mathrm{O}_{2}+\mathrm{O}_{2} \rightarrow \mathrm{HO}_{2}+\mathrm{O}_{2}$ & $1.77 \times 10^{-35} T^{-1.0}$ & 28 \\
\hline 42 & $2 \mathrm{OH}+\mathrm{O}_{2} \rightarrow \mathrm{H}_{2} \mathrm{O}_{2}+\mathrm{O}_{2}$ & $3.5 \times 10^{-35} T^{-0.7}$ & 28 \\
\hline 43 & $\mathrm{H}+\mathrm{O}_{2} \rightarrow \mathrm{OH}+\mathrm{O}$ & $3.3 \times 10^{-13} \exp (-8460 / T)$ & 29 \\
\hline 44 & $\mathrm{H}+\mathrm{H}_{2} \mathrm{O} \rightarrow \mathrm{OH}+\mathrm{H}_{2}$ & $7.5 \times 10^{-19} \exp (-9270 / T)$ & 29 \\
\hline 45 & $\mathrm{H}+\mathrm{H}_{2} \mathrm{O}_{2} \rightarrow \mathrm{H}_{2}+\mathrm{HO}_{2}$ & $2.8 \times 10^{-15} \exp (-1890 / T)$ & 29 \\
\hline 46 & $\mathrm{H}+\mathrm{H}_{2} \mathrm{O}_{2} \rightarrow \mathrm{OH}+\mathrm{H}_{2} \mathrm{O}$ & $1.7 \times 10^{-14} \exp (-1800 / T)$ & 29 \\
\hline 47 & $\mathrm{H}+\mathrm{O}_{2}+\mathrm{H}_{2} \rightarrow \mathrm{HO}_{2}+\mathrm{H}_{2}$ & $5.8 \times 10^{-36} T^{-0.8}$ & 29 \\
\hline 48 & $\mathrm{H}+\mathrm{O}_{2}+\mathrm{H}_{2} \mathrm{O} \rightarrow \mathrm{HO}_{2}+\mathrm{H}_{2} \mathrm{O}$ & $4.3 \times 10^{-36} T^{-0.8}$ & 29 \\
\hline 49 & $\mathrm{H}+\mathrm{OH}+\mathrm{H}_{2} \mathrm{O} \rightarrow \mathrm{H}_{2} \mathrm{O}+\mathrm{H}_{2} \mathrm{O}$ & $3.9 \times 10^{-31} T^{-2.0}$ & 29 \\
\hline 50 & $2 \mathrm{OH}+\mathrm{H}_{2} \mathrm{O} \rightarrow \mathrm{H}_{2} \mathrm{O}_{2}+\mathrm{H}_{2} \mathrm{O}$ & $4.0 \times 10^{-36}$ & 29 \\
\hline 51 & $2 \mathrm{OH} \rightarrow \mathrm{H}_{2} \mathrm{O}+\mathrm{O}$ & $2.9 \times 10^{-23} T^{2.6} \exp (945 / T)$ & 30 \\
\hline 52 & $\mathrm{H}^{+}+\mathrm{O} \rightarrow \mathrm{O}^{+}+\mathrm{H}$ & $3.75 \times 10^{-13}$ & 31 \\
\hline 53 & $\mathrm{H}^{+}+\mathrm{H}_{2} \mathrm{O} \rightarrow \mathrm{H}_{2} \mathrm{O}^{+}+\mathrm{H}$ & $8.2 \times 10^{-12}$ & 31 \\
\hline 54 & $\mathrm{H}^{+}+\mathrm{O}_{2} \rightarrow \mathrm{O}_{2}^{+}+\mathrm{H}$ & $1.17 \times 10^{-12}$ & 31 \\
\hline 55 & $\mathrm{H}_{2}^{+}+\mathrm{H} \rightarrow \mathrm{H}^{+}+\mathrm{H}_{2}$ & $6.4 \times 10^{-13}$ & 31 \\
\hline 56 & $\mathrm{H}_{2}^{+}+\mathrm{H}_{2} \rightarrow \mathrm{H}_{3}^{+}+\mathrm{H}$ & $2.0 \times 10^{-12}$ & 31 \\
\hline 57 & $\mathrm{H}_{2}^{+}+\mathrm{O}_{2} \rightarrow \mathrm{O}_{2}^{+}+\mathrm{H}_{2}$ & $2.7 \times 10^{-12}$ & 31 \\
\hline 58 & $\mathrm{H}_{2}^{+}+\mathrm{H}_{2} \mathrm{O} \rightarrow \mathrm{H}_{2} \mathrm{O}^{+}+\mathrm{H}_{2}$ & $7.3 \times 10^{-12}$ & 31 \\
\hline 59 & $\mathrm{H}_{3}^{+}+\mathrm{O} \rightarrow \mathrm{OH}^{+}+\mathrm{H}_{2}$ & $8.0 \times 10^{-13}$ & 31 \\
\hline 60 & $\mathrm{H}_{3}^{+}+\mathrm{O}_{2} \rightarrow \mathrm{HO}_{2}^{+}+\mathrm{H}_{2}$ & $6.7 \times 10^{-13}$ & 31 \\
\hline 61 & $\mathrm{O}^{+}+\mathrm{H} \rightarrow \mathrm{H}^{+}+\mathrm{O}$ & $6.4 \times 10^{-13}$ & 31 \\
\hline 62 & $\mathrm{O}^{+}+\mathrm{H}_{2} \rightarrow \mathrm{OH}^{+}+\mathrm{H}$ & $1.62 \times 10^{-12}$ & 31 \\
\hline 63 & $\mathrm{O}^{+}+\mathrm{H}_{2} \mathrm{O} \rightarrow \mathrm{H}_{2} \mathrm{O}^{+}+\mathrm{O}$ & $2.6 \times 10^{-12}$ & 31 \\
\hline 64 & $\mathrm{O}^{+}+\mathrm{O}_{2} \rightarrow \mathrm{O}_{2}^{+}+\mathrm{O}$ & $2.1 \times 10^{-14}$ & 31 \\
\hline 65 & $\mathrm{OH}^{+}+\mathrm{H}_{2} \rightarrow \mathrm{H}_{2} \mathrm{O}^{+}+\mathrm{H}$ & $9.7 \times 10^{-13}$ & 31 \\
\hline 66 & $\mathrm{OH}^{+}+\mathrm{H}_{2} \mathrm{O} \rightarrow \mathrm{H}_{2} \mathrm{O}^{+}+\mathrm{OH}$ & $2.89 \times 10^{-12}$ & 31 \\
\hline 67 & $\mathrm{OH}^{+}+\mathrm{O}_{2} \rightarrow \mathrm{O}_{2}^{+}+\mathrm{OH}$ & $3.8 \times 10^{-13}$ & 31 \\
\hline 68 & $\mathrm{H}_{2} \mathrm{O}^{+}+\mathrm{O}_{2} \rightarrow \mathrm{O}_{2}^{+}+\mathrm{H}_{2} \mathrm{O}$ & $3.3 \times 10^{-13}$ & 31 \\
\hline 69 & $\mathrm{HO}_{2}^{+}+\mathrm{H}_{2} \rightarrow \mathrm{H}_{3}^{+}+\mathrm{O}_{2}$ & $3.3 \times 10^{-13}$ & 31 \\
\hline
\end{tabular}


TABLE 3: (continued)

\begin{tabular}{|c|c|c|c|}
\hline No. & Chemical reaction & $\begin{array}{l}\text { Rate constants } \\
\left(\mathrm{m}^{3}-\mathrm{kmol}^{-1}-\mathrm{s}^{-1}\right)\end{array}$ & Ref. \\
\hline 70 & $\mathrm{O}_{3}+\mathrm{O}_{3} \rightarrow \mathrm{O}_{2}+\mathrm{O}+\mathrm{O}_{3}$ & $1.65 \times 10^{-12} \exp (-11435 / T)$ & 32 \\
\hline 71 & $\mathrm{O}_{3}+\mathrm{O}_{2} \rightarrow \mathrm{O}+\mathrm{O}_{2}+\mathrm{O}_{2}$ & $7.26 \times 10^{-13} \exp (-11435 / T)$ & 32 \\
\hline 72 & $\mathrm{O}^{-}+\mathrm{O}_{2} \rightarrow \mathrm{e}+\mathrm{O}_{3}$ & $5.0 \times 10^{-18}$ & 32 \\
\hline 73 & $\mathrm{O}^{-}+\mathrm{O}_{2}+\mathrm{O}_{2} \rightarrow \mathrm{O}_{3}^{-}+\mathrm{O}_{2}$ & $1.0 \times 10^{-35}$ & 32 \\
\hline 74 & $\mathrm{O}_{2}^{-}+\mathrm{O} \rightarrow \mathrm{e}+\mathrm{O}_{3}$ & $3.0 \times 10^{-13}$ & 32 \\
\hline 75 & $\mathrm{O}_{3}^{-}+\mathrm{O}^{+} \rightarrow \mathrm{O}_{3}+\mathrm{O}$ & $1.7 \times 10^{-9} T^{-0.5}$ & 32 \\
\hline 76 & $\mathrm{O}_{3}^{-}+\mathrm{O}_{2}^{+} \rightarrow \mathrm{O}_{3}+\mathrm{O}_{2}$ & $3.5 \times 10^{-9} T^{-0.5}$ & 32 \\
\hline 77 & $\mathrm{O}_{3}^{-}+\mathrm{O} \rightarrow \mathrm{O}_{2}^{-}+\mathrm{O}_{2}$ & $1.1 \times 10^{-14}$ & 32 \\
\hline 78 & $\mathrm{O}^{-}+\mathrm{O}_{3} \rightarrow \mathrm{O}_{3}^{-}+\mathrm{O}$ & $5.5 \times 10^{-13}$ & 32 \\
\hline 79 & $\mathrm{O}^{-}+\mathrm{O}_{3} \rightarrow \mathrm{O}_{2}^{-}+\mathrm{O}_{2}$ & $1.0 \times 10^{-12}$ & 32 \\
\hline 80 & $\mathrm{O}^{-}+\mathrm{O}_{3} \rightarrow \mathrm{e}+2 \mathrm{O}_{2}$ & $3.0 \times 10^{-13}$ & 32 \\
\hline 81 & $\mathrm{O}_{2}^{-}+\mathrm{O}_{3} \rightarrow \mathrm{O}_{3}^{-}+\mathrm{O}_{2}$ & $4.0 \times 10^{-13}$ & 32 \\
\hline 82 & $\mathrm{OH}^{-}+\mathrm{O}_{3} \rightarrow \mathrm{e}+\mathrm{O}_{2}+\mathrm{HO}_{2}$ & $1.0 \times 10^{-15}$ & 32 \\
\hline 83 & $\mathrm{OH}^{-}+\mathrm{O}_{3} \rightarrow \mathrm{O}_{3}^{-}+\mathrm{OH}$ & $5.0 \times 10^{-13}$ & 32 \\
\hline 84 & $\mathrm{OH}^{-}+\mathrm{O}_{3} \rightarrow \mathrm{O}_{2}^{-}+\mathrm{HO}_{2}$ & $1.0 \times 10^{-12}$ & 32 \\
\hline 85 & $2 \mathrm{OH}+\mathrm{O}_{2} \rightarrow \mathrm{H}_{2} \mathrm{O}_{2}+\mathrm{O}_{2}$ & $6.615 \times 10^{-35} T^{-0.8}$ & 34 \\
\hline 86 & $\mathrm{H}_{2} \mathrm{O}^{+}+\mathrm{O}_{2} \rightarrow \mathrm{H}_{2} \mathrm{O}+\mathrm{O}_{2}$ & $3.464 \times 10^{-8} T^{-0.5}$ & 34 \\
\hline 87 & $\mathrm{O}_{2}^{+}+\mathrm{O}_{2}^{-} \rightarrow 2 \mathrm{O}_{2}$ & $3.464 \times 10^{-8} T^{-0.5}$ & 34 \\
\hline 88 & $\mathrm{O}^{+}+\mathrm{O}_{2}^{-} \rightarrow \mathrm{O}+\mathrm{O}_{2}$ & $3.464 \times 10^{-8} T^{-0.5}$ & 34 \\
\hline 89 & $\mathrm{H}+\mathrm{H}_{2} \mathrm{O}_{2} \rightarrow \mathrm{H}_{2} \mathrm{O}+\mathrm{OH}$ & $4.0 \times 10^{-14} \exp (-2000 / T)$ & 34 \\
\hline 90 & $\mathrm{H}+\mathrm{H}_{2} \mathrm{O}_{2} \rightarrow \mathrm{HO}_{2}+\mathrm{H}_{2}$ & $8.0 \times 10^{-14} \exp (-4000 / T)$ & 34 \\
\hline 91 & $\mathrm{H}_{2}+\mathrm{O}_{2} \rightarrow \mathrm{H}+\mathrm{HO}_{2}$ & $2.4 \times 10^{-13} \exp (-28500 / T)$ & 34 \\
\hline 92 & $\mathrm{H}+\mathrm{O}_{2} \rightarrow \mathrm{OH}+\mathrm{O}$ & $2.8 \times 10^{-10} T^{-0.9} \exp (-8750 / T)$ & 34 \\
\hline 93 & $\mathrm{OH}+\mathrm{O}_{2} \rightarrow \mathrm{O}+\mathrm{HO}_{2}$ & $3.7 \times 10^{-14} \exp (-26500 / T)$ & 34 \\
\hline 94 & $\mathrm{OH}+\mathrm{H} \rightarrow \mathrm{O}+\mathrm{H}_{2}$ & $1.14 \times 10^{-15} T^{0.67} \exp (-518 / T)$ & 34 \\
\hline 95 & $\mathrm{HO}_{2}+\mathrm{H}_{2} \rightarrow \mathrm{H}_{2} \mathrm{O}_{2}+\mathrm{H}$ & $5.0 \times 10^{-14} \exp (-13100 / T)$ & 34 \\
\hline 96 & $\mathrm{H}_{2} \mathrm{O}+\mathrm{O} \rightarrow 2 \mathrm{OH}$ & $7.6 \times 10^{-18} T^{1.3} \exp (-8605 / T)$ & 34 \\
\hline 97 & $\mathrm{O}^{-}+\mathrm{H}_{2}^{+} \rightarrow \mathrm{O}+\mathrm{H}_{2}$ & $5.196 \times 10^{-8} T^{-0.5}$ & 34 \\
\hline 98 & $\mathrm{O}_{2}^{-}+\mathrm{H}_{2}^{+} \rightarrow \mathrm{O}_{2}+\mathrm{H}_{2}$ & $5.196 \times 10^{-8} T^{-0.5}$ & 34 \\
\hline 99 & $\mathrm{H}^{-}+\mathrm{H}_{2}^{+} \rightarrow \mathrm{H}+\mathrm{H}_{2}$ & $5.196 \times 10^{-8} T^{-0.5}$ & 34 \\
\hline 100 & $\mathrm{H}^{-}+\mathrm{H}_{2} \mathrm{O}^{+} \rightarrow \mathrm{H}+\mathrm{H}_{2} \mathrm{O}$ & $5.196 \times 10^{-8} T^{-0.5}$ & 34 \\
\hline 101 & $\mathrm{O}^{-}+\mathrm{H}_{2} \mathrm{O}^{+} \rightarrow \mathrm{O}+\mathrm{H}_{2} \mathrm{O}$ & $5.196 \times 10^{-8} T^{-0.5}$ & 34 \\
\hline 102 & $\mathrm{O}^{-}+\mathrm{O}_{2}^{+} \rightarrow \mathrm{O}+\mathrm{O}_{2}$ & $5.196 \times 10^{-8} T^{-0.5}$ & 34 \\
\hline
\end{tabular}




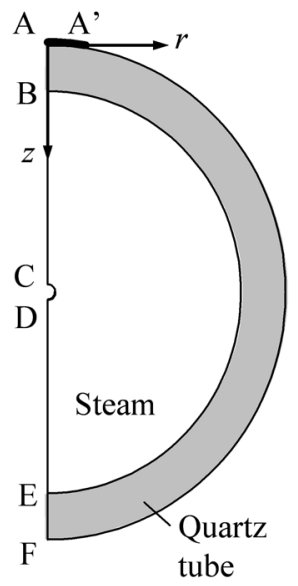

$$
\begin{aligned}
& \mathrm{AB}, \mathrm{EF}: \frac{\partial \phi}{\partial r}=0 \\
& \mathrm{BC}, \mathrm{DE}: v=0, \frac{\partial \phi}{\partial r}=0, \frac{\partial T}{\partial r}=0, \frac{\partial Y}{\partial r}=0 \\
& \overparen{\mathrm{CD}}: \quad \phi=\sum_{n}^{20} \frac{4}{(2 n-1) \pi} \phi_{0} \sin ((2 n-1) \pi \omega t) \\
& \phi_{0}=8 \mathrm{kV}, \omega=4 \mathrm{kHz} \\
& u=v=0, \frac{\partial Y}{\partial n}=0, \quad T=373 \mathrm{~K} \\
& \overparen{\mathrm{BE}}: \quad \varepsilon_{\text {steam }} \frac{\partial \phi_{\text {stem }}}{\partial n}-\varepsilon_{\text {quartz }} \frac{\partial \phi_{\text {quarrz }}}{\partial n}=-\sigma_{s} \\
& \overparen{\mathrm{AA}^{\prime}:} \quad \phi=0 \mathrm{kV}
\end{aligned}
$$

FIG. 9: Boundary conditions.

of $\mathrm{OH}$ between the wire electrode and the wall surface started to decrease, then $\mathrm{OH}$ remained in the vicinity of the wall surface and the wire electrode. The $\mathrm{OH}$ is mainly generated by the electron impact on $\mathrm{H}_{2} \mathrm{O}$ as in equation No. 10 in Table 3, and by the electron-attachment dissociation to $\mathrm{H}_{2} \mathrm{O}^{+}$, shown in equation No. 19 in Table 3. The density of $\mathrm{H}_{2} \mathrm{O}_{2}$ increased gradually in the vicinity of the wire electrode and the wall surface as it possibly decomposed, and it did not decrease because $\mathrm{H}_{2} \mathrm{O}_{2}$ is more stable.

\section{CONCLUSION}

The reactive flow field and the mechanism of sterilization by a steam plasma flow at atmospheric pressure was clarified by experimental and computational methods to develop an innovative autoclave. The results obtained in this study are summarized as follows.

When the applied peak-to-peak voltage was $18 \mathrm{kV}$ with a frequency of $3 \mathrm{kHz}$, sterilization was attained in 40 min for two test samples, although sterilization was observed for only one sample at a peak-to-peak voltage of $16 \mathrm{kV}$. When the air was mixed with the steam flow at a rate of 1 or $4 \mathrm{~L} / \mathrm{min}$, sterilization was attained in 30 minutes for all three test samples, because the $\mathrm{OH}$ generation was increased. The radiation intensity of $\mathrm{OH}$ observed in the discharge region increased with the increase of applied voltage, frequency, and mixing gas flow rate of the air. The $\mathrm{OH}$ radical was converted to $\mathrm{H}_{2} \mathrm{O}_{2}$ in the condensed water. However, the condensed water did not sterilize the spores of G. stearothermophilus. Computational analysis was performed with the axisymmetric, non-Maxwellian model, which clarified that the $\mathrm{OH}$ radicals were generated from the wire electrode toward the ground electrode and that the concentration of $\mathrm{H}_{2} \mathrm{O}_{2}$ increased. 


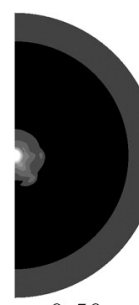

$t=0.50 \mu \mathrm{s}$

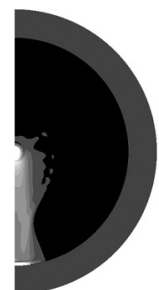

$0.90 \mu \mathrm{s}$

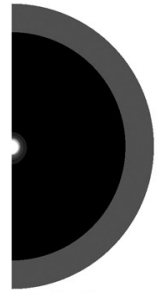

$t=0.50 \mu \mathrm{s}$

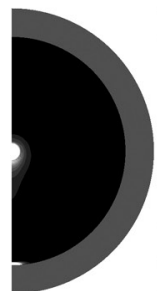

$0.90 \mu \mathrm{s}$

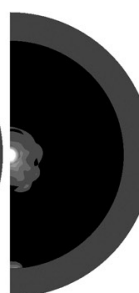

$0.60 \mu \mathrm{s}$

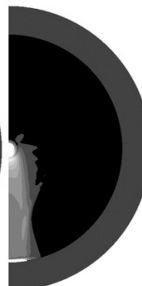

$0.95 \mu \mathrm{s}$

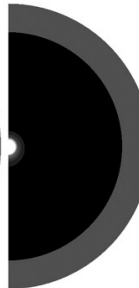

$0.60 \mu \mathrm{s}$

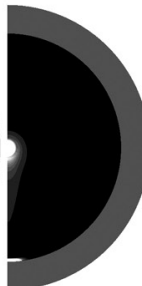

$0.95 \mu \mathrm{s}$

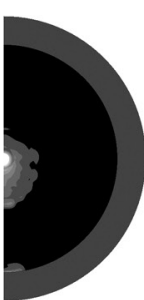

$0.70 \mu \mathrm{s}$

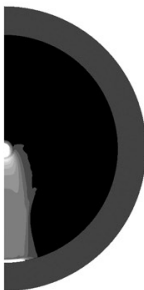

$1.00 \mu \mathrm{s}$

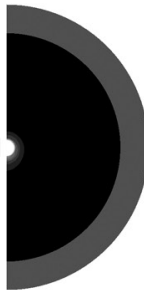

$0.70 \mu \mathrm{s}$

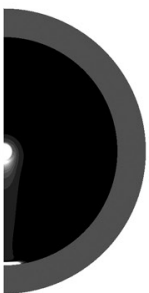

$1.00 \mu \mathrm{s}$

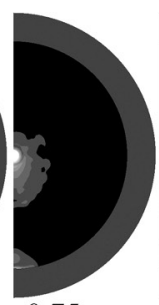

$0.75 \mu \mathrm{s}$

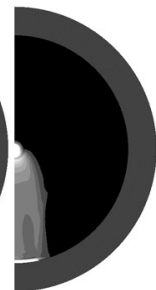

$1.10 \mu \mathrm{s}$

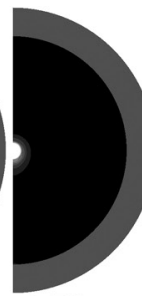

$0.75 \mu \mathrm{s}$

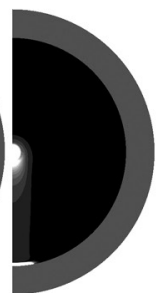

$1.10 \mu \mathrm{s}$

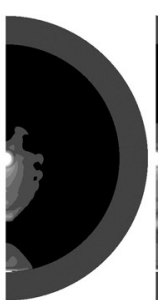

$0.80 \mu \mathrm{s}$

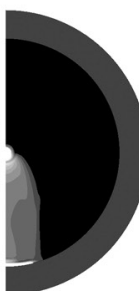

$1.20 \mu \mathrm{s}$

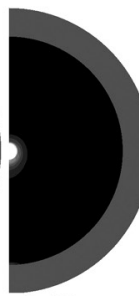

$0.80 \mu \mathrm{s}$

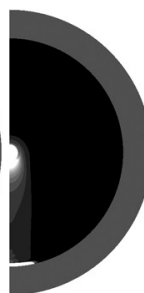

$1.20 \mu \mathrm{s}$

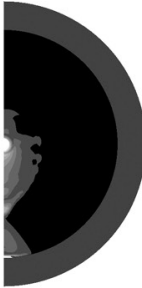

$0.82 \mu \mathrm{s}$

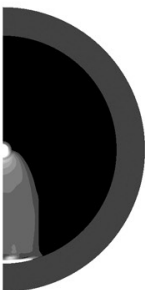

$1.30 \mu \mathrm{s}$

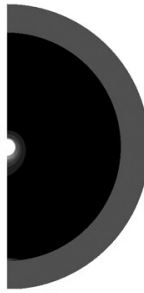

$0.82 \mu \mathrm{s}$

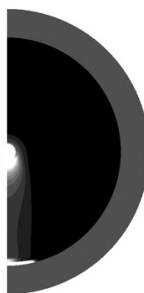

$1.30 \mu \mathrm{s}$

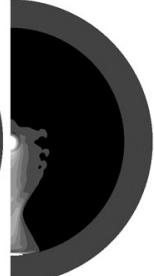

$0.85 \mu \mathrm{s}$

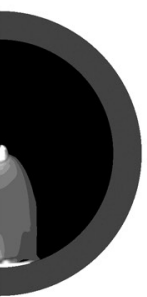

$1.40 \mu \mathrm{s}$

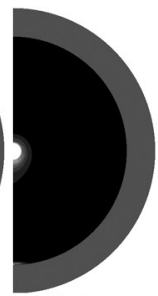

$0.85 \mu \mathrm{s}$

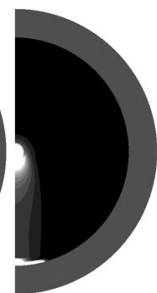

$1.40 \mu \mathrm{s}$ a

$\mathrm{OH}\left(\mathrm{m}^{-3}\right)$

$1 \mathrm{E}+018$

$9 E+017-$
$8 E+017-$

$7 E+017-$

$6 \mathrm{E}+017$

$5 E+017$

$4 \mathrm{E}+017$

$3 E+017-$

$2 E+017$

$1 E+017$

b

$\mathrm{H}_{2} \mathrm{O}_{2}\left(\mathrm{~m}^{-3}\right)$

$2 \mathrm{E}+019$

$1.8 \mathrm{E}+019-$

$1.6 \mathrm{E}+019-$

$1.4 E+019-$

$1.2 E+019-$

$1 \mathrm{E}+019-$

$8 \mathrm{E}+018-$

$6 \mathrm{E}+018-$

$4 \mathrm{E}+018$

$2 \mathrm{E}+018$

0

FIG. 10: Time and spatial evolutions of (a) number density of $\mathrm{OH}$ and (b) number density of $\mathrm{H}_{2} \mathrm{O}_{2}$.

\section{ACKNOWLEDGMENTS}

The authors are grateful to Mr. Igarashi of Hirayama Manufacturing, Japan for helpful discussion. This work was partly supported by a Grant-in-Aid for Scientific Research from the Japan Society for the Promotion of Science, by a Research Grant for Development of Creative Technology Seeds from the Japan Science and Technology Agency, and by a Leadership Research Grant from the Institute of Fluid Science, Tohoku University. 


\section{REFERENCES}

1. Hippler R, Kersten H, Schmidt M, Schoenbach KH, editors. Low temperature plasmas, Vol. 2. Weinheim, Germany: Wiley-VCH; 2008.

2. Fridman G, Friedman G, Gutsol A, Shekhter AB, Vasilets VN, Fridman A. Applied plasma medicine. Plasma Process Polym. 2008;5:503-33.

3. Moisan M, Barbeau J, Moreau S, Pelletier J, Tabrizian M, Yahia L'H. Low-temperature sterilization using gas plasmas: a review of the experiments and an analysis of the inactivation mechanisms. Int J Pharm. 2001;226:1-21.

4. Laroussi M. Nonthermal decontamination of biological media by atmospheric-pressure plasmas: review, analysis, and prospects. IEEE Trans Plasma Sci. 2002;30:1409-15.

5. Shimizu T, Steffes B, Pompl R, Jamitzky F, Bunk W, Ramrath K, Georgi M, Stolz W, Schmidt HU, Urayama T, Fujii S, Morfill GE. Characterization of microwave plasma torch for decontamination. Plasma Process Polym. 2008;5:577-82.

6. Sladek REJ, Stoffels E. Deactivation of Escherichia coli by the plasma needle. J Phys D: Appl Phys. 2005;38:1716-21.

7. Weltmann KD, Brandenburg R, von Woedtke T, Ehlbeck J, Foest R, Stieber M, Kindel E. Antimicrobial treatment of heat sensitive products by miniaturized atmospheric pressure plasma jets (APPJs). J Phys D: Appl Phys. 2008;41:194008.

8. Deng XT, Shi JJ, Shama G, Kong M. Effects of microbial loading and sporulation temperature on atmospheric plasma inactivation of Bacillus subtilis spores. Appl Phys Lett. 2005;87:153901.

9. Moisan M, Barbeau J, Crevier MC, Pelletier J, Philip N, Saoudi B. Plasma sterilization. Methods and mechanisms. Pure Appl Chem. 2002;74:349-58.

10. Laroussi M, Leipold F. Evaluation of the roles of reactive species, heat, and UV radiation in the inactivation of bacterial cells by air plasmas at atmospheric pressure. Int J Mass Spectrom. 2004;233:81-6.

11. Kim GC, Kim GJ, Park SR, Jeon SM, Seo HJ, Iza F, Lee JK. Air plasma coupled with antibody-conjugated nanoparticles: a new weapon against cancer. J Phys D: Appl Phys. 2009;42:032005.

12. Sato T, Miyahara T, Doi A, Ochiai S, Urayama T, Nakatani T. Sterilization mechanism for Escherichia coli by plasma flow at atmospheric pressure. Appl Phys Lett. 2006;89:073902.

13. Sato T, Doi A, Urayama T, Nakatani T, Miyahara T. Inactivation of Escherichia coli by a coaxial microwave plasma flow. IEEE Trans Ind Appl. 2007;43:1159-63.

14. Sato T, Furuya O, Ikeda K, Nakatani T. Generation and transportation mechanisms of chemically active species by dielectric barrier discharge in a tube for catheter sterilization. Plasma Process Polym. 2008;5:606-14.

15. Sato T, Furuya O, Nakatani T. Characteristics of nonequilibrium plasma flow and its sterilization efficacy in a tube at atmospheric pressure. IEEE Trans Ind Appl. 2009;45:44-9.

16. Furui T, Sato T. Sterilization efficacy of steam plasma flow at atmospheric pressure. Trans. Japan Society Mech. Eng. B. 2008;70:879. [in Japanese]

17. Okubo M, Kuroki T, Miyairi Y, Yamamoto T. Low-temperature soot incineration of diesel particulate filter using remote nonthermal plasma induced by a pulsed barrier discharge. IEEE Trans Ind Appl. 2004;40:1504-12.

18. Sato T, Ochiai S, Urayama T. Generation and transport mechanisms of chemical species by a post-discharge flow for inactivation of bacteria. New J Phys. 2009;11:115018. 
19. Miyahara T, Ochiai S, Sato T. Interaction mechanism between a post-discharge flow and water surface. EPL. 2009;86:45001.

20. Sato T, Fujioka K, Ramasamy R, Urayama T, Fujii S. Sterilization efficacy of a coaxial microwave plasma flow at atmospheric pressure. IEEE Trans Ind Appl. 2006;42:399-404.

21. Pearse RWB, Gaydon AG. The identification of molecular spectra. New York: John Wiley \& Sons; 1950.

22. NIST Atomic spectra database [database on the Internet]. Available from: http://physics. nist.gov/PhysRefData/ASD/index.html.

23. Itikawa Y, Mason N. Cross sections for electron collisions with water molecules. J Phys Chem Ref Data. 2005;34:1-22.

24. NIST: electron-impact ionization cross section database [database on the Internet]. Available from: http://www.nist.gov/pml/data/ionization/index.cfm.

25. Atkinson R, Baulch DL, Cox RA, Crowley JN, Hampson RF, Hynes RG, Jenkin ME, Rossi MJ, Troe J. Evaluated kinetic and photochemical data for atmospheric chemistry: Volume I - gas phase reactions of Ox, HOx, NOx and SOx species. Atmos Chem Phys. 2004;4:1461738.

26. Cohen N, Westberg KR. Chemical kinetic data sheets for high temperature reactions. Part I. J Phys Chem Ref Data. 1983;12:531-90.

27. Baulch DL, Cox RA, Hampson Jr. RF, Kerr JA, Troe J, Watson RT. Evaluated kinetic and photochemical data for atmospheric chemistry, CODATA Task Group on Chemical Kinetics. J Phys Chem Ref Data. 1980;9:295-471.

28. Baulch DL, Cox RA, Crutzen PJ, Hampson Jr. RF, Kerr JA, Troe J, Watson RT. Evaluated kinetic and photochemical data for atmospheric chemistry: Supplement I, CODATA Task Group on Chemical Kinetics. J Phys Chem Ref Data. 1982;11:327-496.

29. Baulch DL, Cobos CJ, Cox RA, Esser C, Frank P, Just Th., Kerr JA, Pilling MJ, Troe J, Walker RW, Warnatz J. Evaluated kinetic data for combustion modelling. J Phys Chem Ref Data. 1992;21:295411.

30. Atkinson R, Baulch DL, Cox RA, Crutzen PJ, Hampson Jr. RF, Kerr JA, Rossi MJ, Troe J. Evaluated kinetic and photochemical data for atmospheric chemistry: Supplement VI. IUPAC Subcommittee on Gas Kinetic Data Evaluation for Atmospheric Chemistry. J Phys Chem Ref Data. 1997;26:1329-500.

31. Anicich VG. Evaluated bimolecular ion-molecule gas phase kinetics of positive ions for use in modeling planetary atmospheres, cometary comae, and interstellar clouds. J Phys Chem Ref Data. 1993;22:1469-570.

32. Steinfeld JI, Adler-Golden SM, Gallagher JW. Critical survey of data on the spectroscopy and kinetics of ozone in the mesosphere and thermosphere. J Phys Chem Ref Data. 1987;16:911-52.

33. Rowe BR, Vallee F, Queffelec JL, Gomet JC, Morlais M. The yield of oxygen and hydrogen atoms through dissociative recombination of $\mathrm{H}_{2} \mathrm{O}+$ ions with electrons. J Chem Phys. 1988;88:845-50.

34. Tsang W, Hampson RF. Chemical kinetic data base for combustion chemistry. Part I. Methane and related compounds. J Phys Chem Ref Data. 1986;15:1087-222.

35. CFD-ACE+ V2008.0 Modules Manual V2. ESI-Group; 2008. 